\title{
Identifying targetable genetic features in primary CNS lymphoma
}

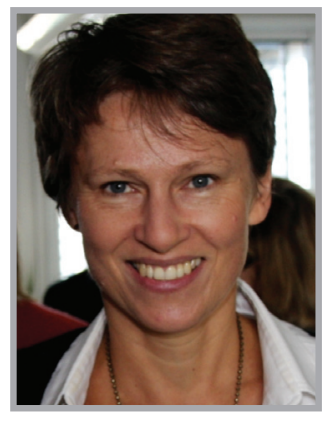

\author{
"Using whole-genome sequencing and target \\ sequencing, genomic alterations typical for the \\ activated B-cell-type diffuse large B-cell lymphoma \\ were found in primary CNS lymphoma..."
}

Agnieszka Korfel $^{*, 1} \&$ Uwe Schlegel $^{2}$

First draft submitted: 1 November 2016; Accepted for publication: 21 November 2016; Published online: 13 January 2017

Primary CNS lymphoma (PCNSL) is a rare variant of malignant lymphoma accounting for $2-4 \%$ of intracranial neoplasms and $4-6 \%$ of extranodal lymphomas. The vast majority of PCNSLs are diffuse large B-cell lymphoma (DLBCL) morphologically indistinguishable from nodal or other extranodal DLBCL [1]. The pathogenesis of PCNSL is not completely understood, however, the high rate of somatic hypermutations in PCNSL tumor cells suggests that the initial transformation event in PCNSL tumor cells occurs outside the CNS, because this requires the influence of a microenvironment present in germinal centers of secondary lymphatic organs [2].

PCNSL is a diffuse brain disease which cannot be treated adequately with local therapies alone. Systemic chemotherapy is the most important treatment but to be effective, drugs need to cross the intact blood-brain barrier. High-dose methotrexate $\left(\geq 3 \mathrm{~g} / \mathrm{m}^{2}\right)$ based chemotherapy represents the current standard of treatment in PCNSL. The most recent therapeutic concepts include intensive high-dose methotrexate based polychemotherapy combined with the anti-CD20 antibody, rituximab for induction followed by consolidation high-dose chemotherapy with autologous stem cell transplantation or noncross-resistant 'conventional' chemotherapy. Whole-brain radiotherapy as part of initial treatment is rather avoided. With this treatment, 2-year progressionfree survival (PFS) of $61-82 \%$ and 2 -year reported [3-5]. However, these results have been achieved in relatively small studies with selected, mostly younger patients without significant comorbidity, with the survival curves at longer follow-up lacking a plateau and thus indicating that patients continue to relapse with only a minority probably being cured [6]. Results in elderly patients are even poorer [7]. Refractory/relapsed ( $\mathrm{r} / \mathrm{r})$ patients have a very poor prognosis, particularly those with disease refractory to first-line therapy and short duration of first remission [8]. overall survival of $69-80 \%$ has been

\section{KEYWORDS}

- blood-brain barrier • target therapy $\bullet$ toxicity 
"Therefore, more effective and less toxic treatments are needed in primary CNS lymphoma."
Toxicity of current therapies is an additional problem. Mortality rates on therapy can reach $10 \%$, and may exceed this number in the elderly. This group is also at major risk for delayed CNS toxicity, particularly when whole-brain radiotherapy is administered [1].

Therefore, more effective and less toxic treatments are needed in PCNSL. Novel agents targeting important survival pathways of tumor cells or their microenvironment could represent such an option. Due to the paucity of the disease, studies evaluating the molecular landscape as a basis for targeted therapies are rare in PCNSL as compared with systemic lymphoma. Moreover, their results are frequently difficult to interpret due to small cohort sizes and lack of paired normal samples. Using whole-genome sequencing and target sequencing, genomic alterations typical for the activated B-cell (ABC)-type DLBCL were found in PCNSL with frequent mutations of key regulators of the Toll-like receptor (TLR)/B-cell receptor (BCR)/mTOR signaling pathway. MYD88 and CD79B mutations were found in $76-86 \%$ and $64-83 \%$, respectively and mutations of CARD11 in 16-29\% of cases [2,9-14]. As a result, the NF- $\kappa B$ pathway is activated. Numerous target drugs aiming at this signaling pathway are available.

The allosteric inhibitor of mTOR, temsirolimus was the first target drug evaluated in a formal Phase II trial in PCNSL [15]. In heavily pretreated $r / r$ patients $(n=37)$ with a median age of 70 years, a high-remission rate of 54\% (complete-response rate 20.5\%) was found at salvage with temsirolimus monotherapy. However, median PFS was 2.1 months only, and toxicity was considerable with grade 3-4 infections in $19 \%$ of patients and death on therapy in $13.5 \%$. Interestingly, temsirolimus was detectable in the cerebrospinal fluid (CSF) in only one of nine patients evaluated. This discrepancy between obvious therapeutic activity and lack of detectable concentration in the CSF is not clear and might be the result of a better penetration to brain parenchyma as into the CSF.

Temsirolimus inhibits mTOR within the TORC1 complex with activation of TORC2 proposed as a putative resistance mechanism. A simultaneous inhibition of PI3K could synergistically reduce subsequent protein kinase B (AKT) activation, which potentially could enable tumor cells to bypass mTOR inhibition. Such a synergistic mode of action is currently being evaluated in a Phase I/II study with the
pan-PI3K, mTORC1 and mTORC2 inhibitor, PQR309 (PIQUR, Basel, Switzerland, NCT02669511). Importantly, in pre-clinical animal models PQR309 has been demonstrated to penetrate the blood-brain barrier at pharmacodynamically active concentrations.

Ibrutinib, an inhibitor of the bruton tyrosine kinase positioned early within the BCR-signaling cascade and linking the BCR and the NF- $\mathrm{BB}$ pathway, is a potentially attractive candidate to be evaluated in PCNSL regarding its effectivity in ABC-DLBCL and its assumed CNS penetration due to small size [16]. In fact, rapid response to single-agent ibrutinib has been seen in all three patients with CNS relapse of mantle cell lymphoma without severe toxicity. Remarkably, CSF penetration of ibrutinib was demonstrated with a CSF-to-plasma concentration ratio of 1-7\% [17]. Consequently, single-agent ibrutinib is now being evaluated in a Phase I trial (NCT02315326) for patients with $\mathrm{r} / \mathrm{r}$ PCNSL and secondary CNS lymphoma. Preliminary results are promising: seven of nine evaluated patients responded; the median PFS was 6 months [18]. Moreover, treatment has been well tolerated with two grade 4 toxicities (neutropenia and lymphopenia) that resolved after the drug was held.

Ibrutinib is an immunosuppressive drug and in combination with other lymphotoxic drugs, particularly dexamethasone, which is frequently used in PCNSL, exposes patients to a high risk of live-threatening infections. This is impressively illustrated by a study (NCT02203526) combining ibrutinib with classic chemo-/immunotherapy (temozolomide, etoposide, doxil, rituxi$\mathrm{mab}$ ), dexamethasone and intraventricular cytarabine (DA-TEDDI-R). 15 of 18 patients responded, most likely due to the fact that both $\mathrm{r} / \mathrm{r}$ PCNSL and newly diagnosed patients had been included. Moreover, a high CSF/plasma ratio of $21.4-100 \%$ for ibrutinib and $48-120 \%$ for its metabolite were found. However, the study had to be stopped since four patients developed definite and three possible invasive aspergillosis, two of which were lethal [19].

Considering the selective tropism of PCNSL to the brain, the microenvironment likely plays a role in its pathogenesis and survival. The interaction between PD-L1 on tumor cells and PD-1 on TIL and tumor-associated macrophages (TAM) could be one of the possible mechanisms used by PCNSL to create immmunotolerance of the surrounding tissue. Immunohistologic studies revealed expression of PD-L1 or PD-1 on tumor 
cells, TIL or TAM in 18/20 (90\%) of PCNSL cases; $12 / 20(60 \%)$ of cases showed positivity of TIL for PD-1+ and in 4/20 (20\%) of cases positivity of TAM for PD-L1+ [20]. Molecular analyses confirmed the frequent exhibition of 9p24.1/PD-L1/PD-L2 copy number alterations and translocations in PCNSL [14]. Based on these results, inhibition of PD-1 signaling by boosting antitumor immune responses may represent a promising option for target therapy in PCNSL. Since PD-1 activity is induced not only locally in the tumor but also systemically, the blood-brain barrier is not expected to interfere significantly with check-point inhibitors activity. Preliminary results are promising: response has been achieved in all four patients with $r / r$ PCNSL treated with the anti-PD-1 antibody, nivolumab; three patients were progression-free at $\geq 6$ months. No patients have had $\geq$ grade 3 toxicities [21]. In an ongoing study, another anti-PD-1 antibody, pembrolizumab is being evaluated in $r / r$ PCNSL (NCT02779101).

The immunomodulatory imide drugs, which act by modifying the tumor microenvironment and activating cytotoxic $\mathrm{T}$ - and natural killer cells, and indirectly inhibiting NF- $\mathrm{BB}$ signaling, showed efficiency in ABC-DLBCL. In a retrospective series, four of six patients with $\mathrm{r} / \mathrm{r}$ PCNSL responded to lenalidomide (one patient for $24+$ months) with no grade 3-4 toxicity [22]. Lenalidomide combined with rituximab is now being evaluated in a Phase I-study (NCT01542918) with 13 patients (8 PCNSL, 5 secondary CNS lymphoma) having been reported [23]. Response was achieved in eight, of whom two maintained complete response $>1.8$ years. In this report, an independent cohort of 12 patients with recurrent disease who received lenalidomide $(5-10 \mathrm{mg})$ as a maintenance after salvage is included: five stayed in remission for $>2$ years. Trough lenalidomide concentration in ventricular CSF was highest at $20 \mathrm{mg}$ dose. In another Phase I trial, pomalidomide combined with dexamethasone is being tested in $\mathrm{r} / \mathrm{r}$ PCNSL or intraocular lymphoma (NCT01722305).

As the insight into the molecular pathogenesis of PCNSL grows, an increasing number of targets for specific therapy are currently being identified in PCNSL. As summarized above, most targets currently addressed within clinical trials are aimed at the TLR/BCR/NF- $\mathrm{KB}$ pathway or at the (immunosuppressive) microenvironment of the tumors. Although the effectivity of some target drugs has been demonstrated, severe unexpected toxicity has also been observed, particularly with drug combinations. PCNSL patients are subjected to an aggressive disease, but in general they are also frailer as compared with patients with systemic DLBCL and frequently immunosuppressed by steroids. Therefore, caution is mandatory while searching for therapeutic progress, and monitoring for toxicity is a pivotal condition.

\section{Financial \& competing interests disclosure}

A Korfel received honoraria from PIQUR, Mundipharma and Riemser. The authors have no other relevant affiliations or financial involvement with any organization or entity with a financial interest in or financial conflict with the subject matter or materials discussed in the manuscript apart from those disclosed.

No writing assistance was utilized in the production of this manuscript.

\section{References}

1 Korfel A, Schlegel U. Diagnosis and treatment of primary CNS lymphoma. Nat. Rev. Neurol. 9(6), 317-327 (2013).

2 Fukumura K, Kawazu M, Kojima $S$ et al. Genomic characterization of primary central nervous system lymphoma. Acta Neuropathol. 131(6), 865-875 (2016).

3 Rubenstein JL, Hsi ED, Johnson JL et al. Intensive chemotherapy and immunotherapy in patients with newly diagnosed primary CNS lymphoma: CALGB 50202 (Alliance 50202). J. Clin. Oncol. 31(25), 3061-3068 (2013).

4 Omuro A, Correa DD, DeAngelis LM et al. R-MPV followed by high-dose chemotherapy with TBC and autologous stem-cell transplant for newly diagnosed primary CNS lymphoma. Blood 125(9), 1403-1410 (2015).

5 Illerhaus G, Kasenda B, Ihorst G et al. High-dose chemotherapy with autologous haemopoietic stem cell transplantation for newly diagnosed primary CNS lymphoma: a prospective, single-arm, Phase II trial. Lancet Haematol. 3(8), e388-e397 (2016).

6 Kasenda B, Schorb E, Fritsch K, Finke J, Illerhaus G. Prognosis after high-dose chemotherapy followed by autologous stem-cell transplantation as first-line treatment in primary CNS lymphoma-a long-term follow-up study. Ann. Oncol. 26(3), 608-611 (2015).
7 Roth P, Martus P, Kiewe P et al. Outcome of elderly patients with primary CNS lymphoma in the G-PCNSL-SG-1 trial. Neurology 79(9), 890-896 (2012).

8 Langner-Lemercier S, Houillier C, Soussain C et al. Primary CNS lymphoma at first relapse/ progression: characteristics, management, and outcome of 256 patients from the French LOC network. Neuro Oncol. 18(9), 1297-1303 (2016).

9 Gonzalez-Aguilar A, Idbaih A, Boisselier B et al. Recurrent mutations of MYD88 and TBL1XR1 in primary central nervous system lymphomas. Clin. Cancer Res. 18(19), 5203-5211 (2012). 


\section{EDITORIAL Korfel \& Schlegel}

10 Deckert M, Montesinos-Rongen M, Brunn A, Siebert R. Systems biology of primary CNS lymphoma: from genetic aberrations to modeling in mice. Acta Neuropathol. 127(2), 175-188 (2014).

11 Bruno A, Boisselier B, Labreche $\mathrm{K}$ et al. Mutational analysis of primary central nervous system lymphoma. Oncotarget 5(13), 5065-5075 (2014).

12 Vater I, Montesinos-Rongen M, Schlesner M et al. The mutational pattern of primary lymphoma of the central nervous system determined by whole-exome sequencing. Leukemia 29(3), 677-685 (2015).

13 Nakamura T, Tateishi K, Niwa T et al. Recurrent mutations of CD79B and MYD88 are the hallmark of primary central nervous system lymphomas. Neuropathol. Appl. Neurobiol. 42(3), 279-290 (2016).

14 Chapuy B, Roemer MG, Stewart C et al. Targetable genetic features of primary testicular and primary central nervous system lymphomas. Blood 127(7), 869-881 (2016).
15 Korfel A, Schlegel U, Herrlinger U et al. Phase II trial of temsirolimus for relapsed/ refractory primary CNS lymphoma. J. Clin. Oncol. 34(15), 1757-1763 (2016).

16 Wilson WH, Young RM, Schmitz R et al. Targeting $B$ cell receptor signaling with ibrutinib in diffuse large B cell lymphoma. Nat. Med. 21(8), 922-926 (2015).

17 Bernard S, Goldwirt L, Amorim S et al. Activity of ibrutinib in mantle cell lymphoma patients with central nervous system relapse. Blood 126(14), 1695-1698 (2015).

18 Grommes C, Kaley T, Nolan C et al. Phase I study of single agent ibrutinib in recurrent/ refractory primary (PCNSL) and secondary CNS lymphoma (SCNSL). Presented at: ASCO Annual Meeting. Chicago, IL, USA, 3-7 June 2016.

19 McGinley L. National Cancer Institute researcher was months late in notifying authorities about deaths. The Washingtton Post, 21 October (2016).
20 Berghoff AS, Ricken G, Widhalm G et al. PD1 (CD279) and PD-L1 (CD274, B7H1) expression in primary central nervous system lymphomas (PCNSL). Clin. Neuropathol. 33(1), 42-49 (2014).

21 Nayak L, Iwamoto F. Treatment of recurrent primary central nervous system lymphoma patients with anti-PD1 antibody nivolumab. Presented at: $68^{\text {th }}$ Annual Meeting of American Academy of Neurology. Vancouver, Canada, 16-21 April 2016.

22 Houillier C, Choquet S, Touitou V et al. Lenalidomide monotherapy as salvage treatment for recurrent primary CNS lymphoma. Neurology 84(3), 325-326 (2015).

23 Rubenstein J, Fraser E, Formaker P et al. Phase I investigation of lenalidomide plus rituximab and outcomes of lenalidomide maintenance in recurrent CNS lymphoma. Presented at: The American Society of Clinical Oncology annual meeting, Chicago, IL, USA, 3-7 June 2016. 